\title{
Design Analog Buffering on The multiplexer in RFID sensor tag with CMOS Technology $0.35 \mu \mathrm{m}$
}

\author{
Dyah Nur'ainingsih, Hamzah Afandi, Eri Prasetyo Wibowo and Busono Soerowirdjo \\ Universitas Gunadarma, J1. Margonda Raya 100 Depok, Jawa Barat, Indonesia
}

\begin{abstract}
This paper describes the 'buffering system in the RFID sensor tags'. Buffer circuit function is used in the data selection as a buffer. Two-sensor output which is an analog signal by a multiplexer owns a possibility of a very low value. The buffer circuit is required to maintain the signal in order to prevent the dropped voltage. Its uses two inverters functioned as a noninverting amplifier. The conclusion is that the buffering circuit is able to keep the voltage level by amplification of 1 .
\end{abstract}

Keywords-RFID sensor tags; multiplexer; water level sensor; temperature sensor; buffer

\section{INTRODUCTION}

RFID is an identification technology that is flexible, easy to use and suitable for the automatic operating system. RFID is available in read-only devices, or that can be read and written (Read/Write). RFID is also one form of auto-ID technologies, which are the object identification technology using radio frequency to read information from an information transmitter device called RFID tags or transponders (Transmitter + Responder).

RFID is a method that can be used to store or receive data remotely using a device called RFID tags or transponders. An RFID tag is a small object, such as an adhesive sticker, and can be attached to an item or product. [1]
The references are taken from Muralikrishna Sathyamurthy's research results, and a team with the title "UVM-based verification methodology for RFID-enabled smart-sensor systems", published in the Analog Integrated Circuits and Signal Processing, January 2014, volume 78, the US Springer publisher. [2]. Muralikrishna utilizes smartsensor-based RFID with mixed-signal system used for bio analytical application. In this paper, the ADC and sensor embedded in the RFID have not been developed or designed yet.

Therefore, this study aims to design the RFID sensor tag planted in a plant seeding media and used to determine water level and temperature around the plant seeds. RFID tag designed requires ADC which functions to convert the analog data signal from the sensor into digital data signal. In addition, it requires a temperature sensor and a water level sensor embedded in RFID tags. The data signal transmitted is based on the requirements. Therefore, a digital selector or multiplexer is needed. The multiplexer works based on a control from the control section that uses back scattering. For more details, it can be seen in Figure 1. So far, the researcher has made a multiplexer circuit as a data selection with data signal input from the sensor and the output is data signals selected.

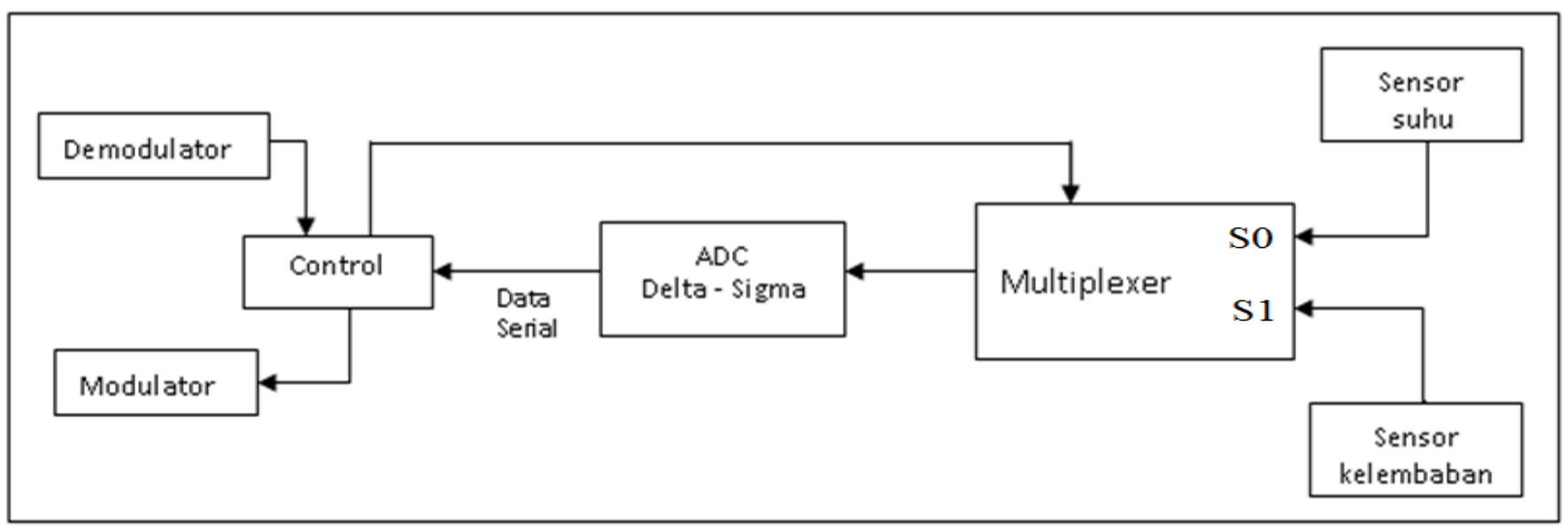

FIGURE I. BLOCK DIAGRAM OF EMBEDDED SENSOR SYSTEM OF RFID TAG

\section{SENSOR SYSTEM}

According to [3], sensor is a device that converts a physical phenomenon into electrical signal. Thus, the sensor is a part of the interface between the physical world and the world of electrical device, such as computer. Another part of this interface is represented by an actuator, which converts electrical signals into physical phenomena. 
Sensor is a transducer that is used to detect a conditions of a process. A transducer is a hardware to convert the information of an energy form into another form proportionally. For instance, sensor for measuring the fuel level in a tank car. The amount of level or position is converted into a transducer signal on the car dashboard into a resistance quantity then converted into an electrical quantity to be displayed .

Sensor is a device used for detecting symptoms or signals derived from a change of energy such as electric energy, physical energy, chemical energy, biological energy, mechanical energy and so on. [4]

The transducer is a device when driven by an energy in a transmission system, will transmit the energy in the same form or in a different form to the next transmission system". The energy transmission can be electrical, mechanical, chemical, optical (radiation) or thermal (heat ). [5]

Some important characteristics of the sensor performance is a transfer function, sensitivity, dynamic range, accuracy or precision, linearity, resolution and bandwidth.

\section{MultiPleXER}

The multiplexer is a logic circuit which receives some data input and chooses one of these inputs at a certain moment, to be issued. The multiplexer circuit designed is a circuit of 2:1 NMOS or also called data selector circuit. [6]

A 2:1 multiplexer is a basic block of "logical switch". The concept of the logical switch is that the logical circuit is implemented as a combination of switches of logic gates. The circuit is matched to the need for choosing the data signal between the temperature sensor or water level sensor. The data selector circuit can be seen as in figure 2 .

$\mathrm{S} 0$ and $\mathrm{S} 1$ is a water level sensor and a temperature sensor. While port $a$ is a signal for water level sensor and port $b$ is the data signal for the temperature sensor. If the control section requests data from the temperature sensor, the water level sensor is disabled or is not selected, and vice versa. For more details, theoutput signal result of the multiplexer circuit or the data selector is shown in figure 3.

Initial problem arising is the signal of the selector dataresult continuously transmitted, so that when the delta sigma ADC must convert an analog data into a digital data, it takes time and impedance potentially happens. Can be seen in figure 3 , the signal "V (output) " is an output multiplexer with a voltage level approaching 1.5 volts. Then the signal "V (out)" is an output from the buffer circuit slightly below 1.45 volts. This proves that the buffering is able to maintain the data signal in order little bit to drop. Thus amplification of the buffer is limit 1. So, if the impedance happens in the sigma-delta ADC, the data signal does not drop.

There are six signals which the outcome is observed, e.i. S0 is a water level sensor, $\mathrm{A}$ is the data signal from water level sensor. S1 is the temperature sensor and B is the data signal of the temperature sensor. The OUTPUT signal is the output or data selected. The OUT signalis the output of buffer circuit. Mean while, the layout of the multiplexer circuit $2: 1$ is in Figure 3 . The actual size is $22 \mu \mathrm{m}$ for the length and $13 \mu \mathrm{m}$ for the width.

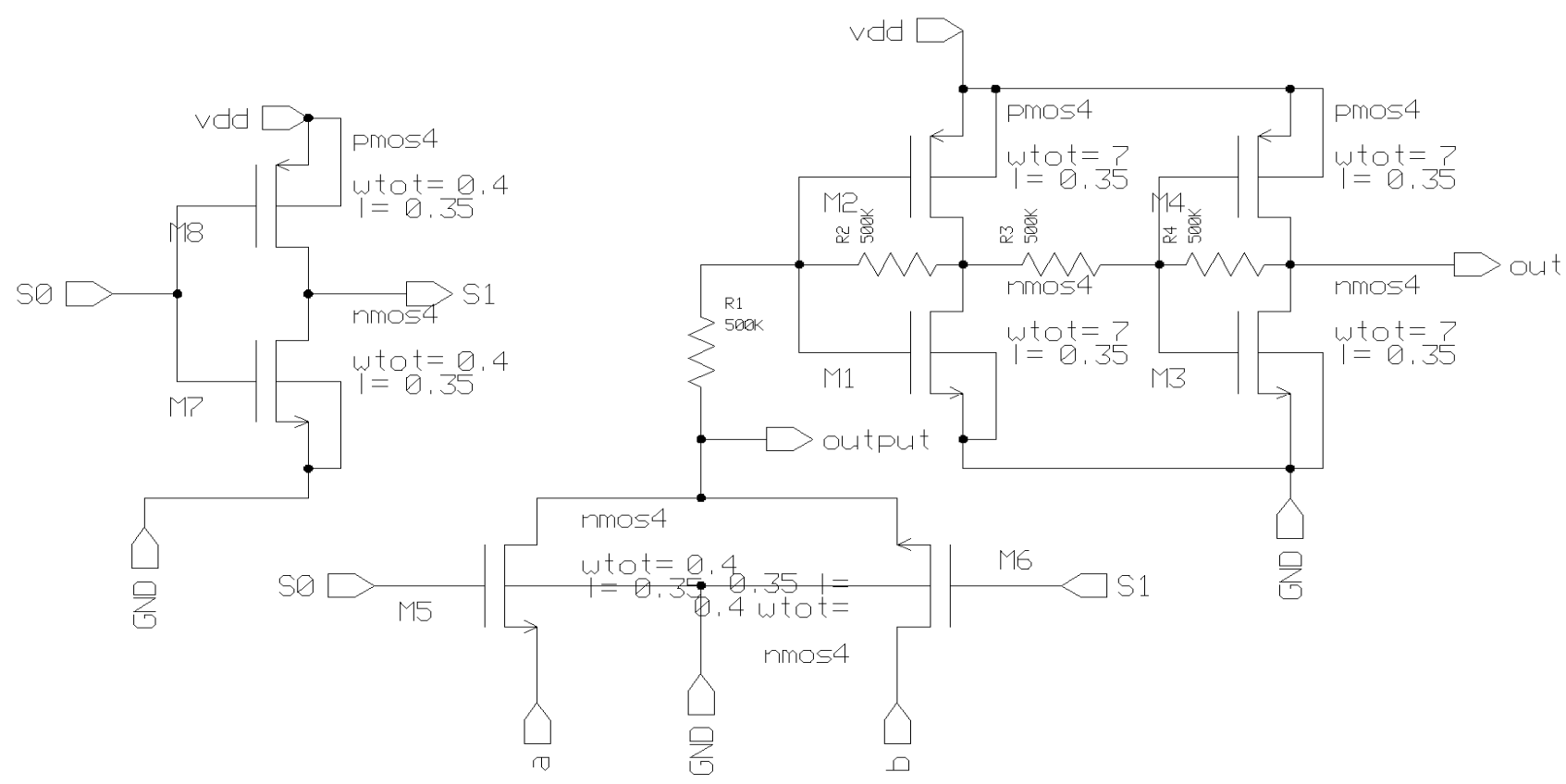

FIGURE II. CIRCUIT OF MULTIPLEXER 2:1 

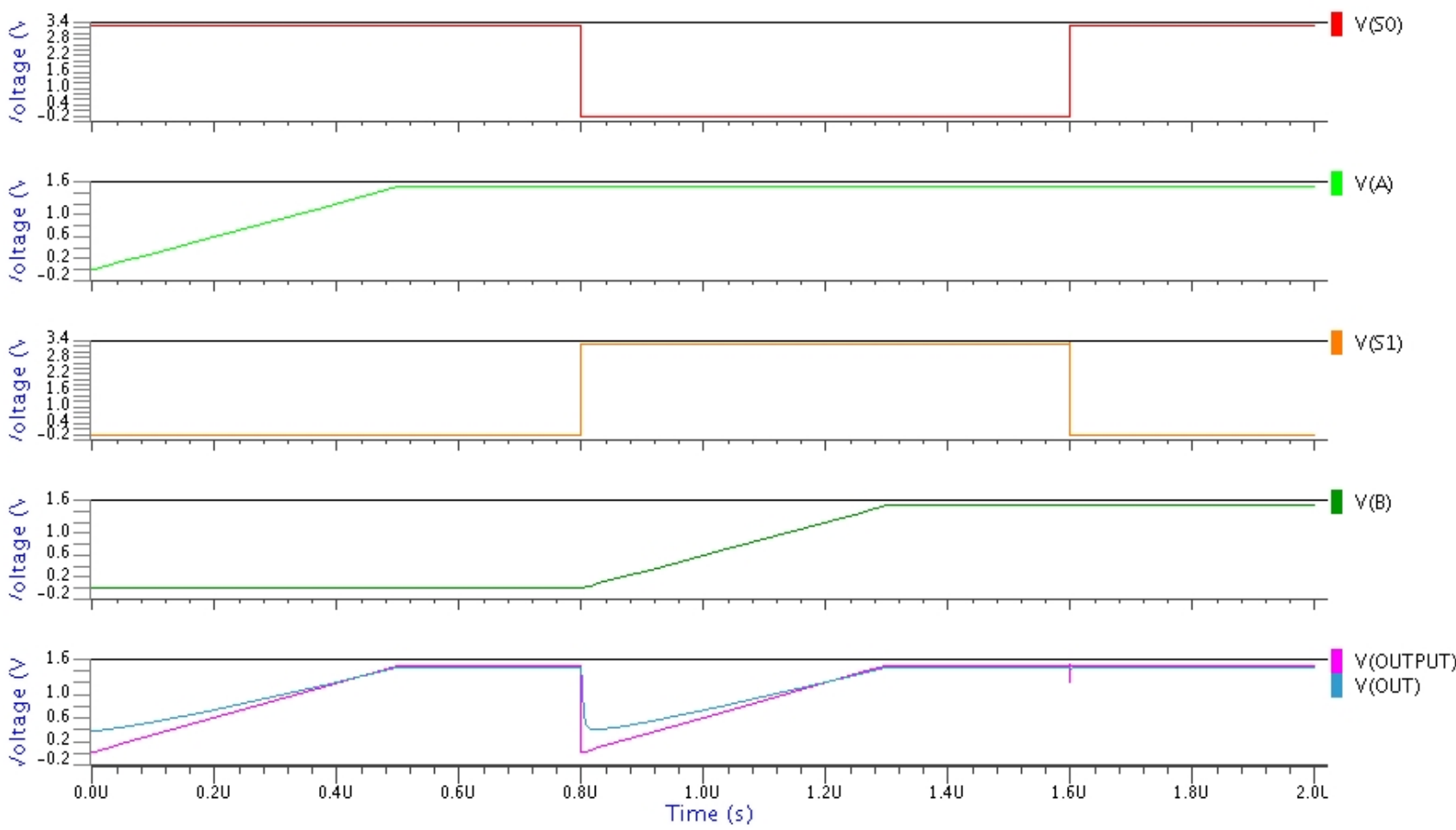

FIGURE III. OUTPUT SIGNAL OF $2: 1$

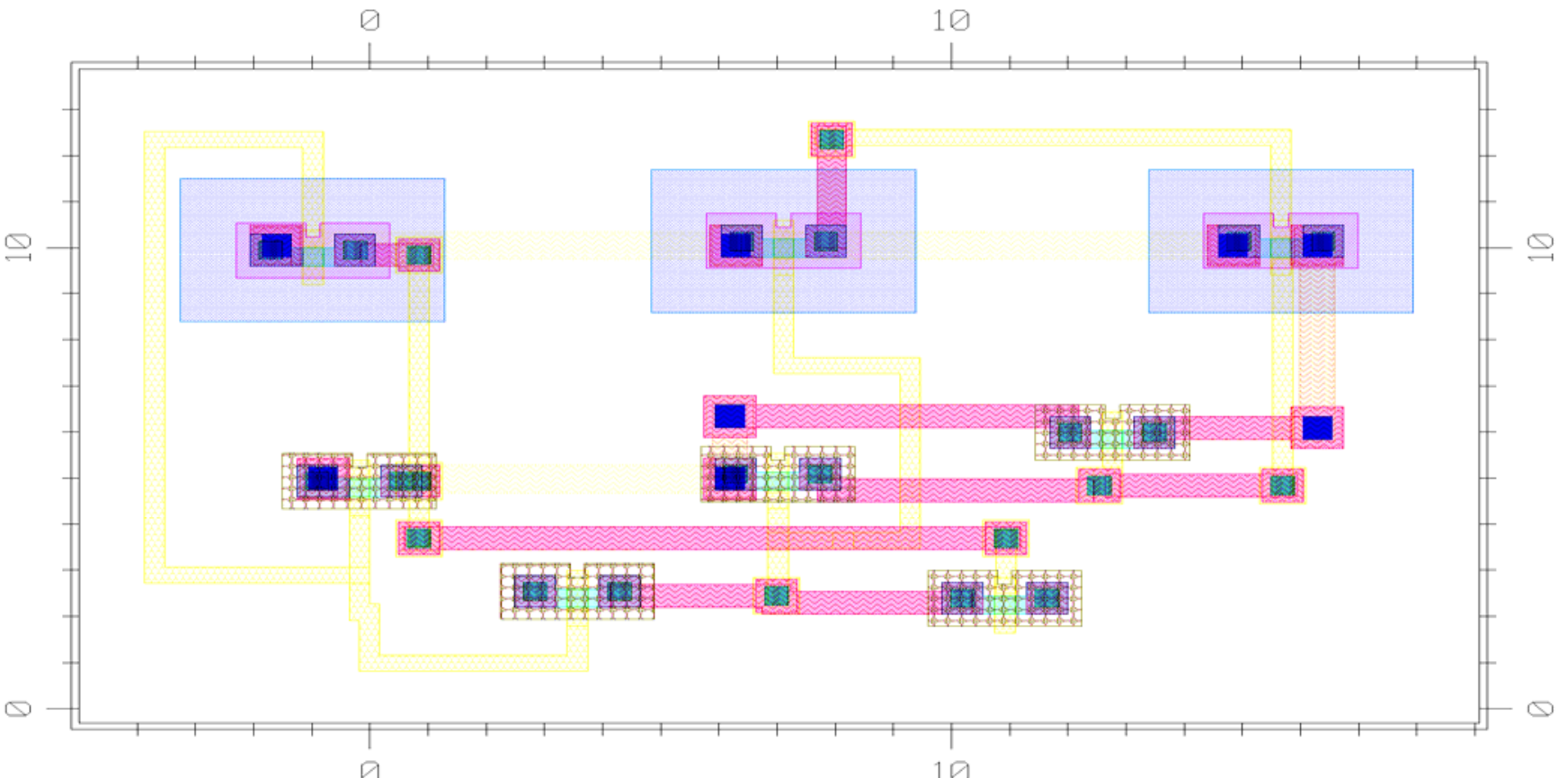

FIGURE IV. LAYOUT OF $2: 1$ MULTIPLEKXER 


\section{CONCLUSION}

The transfer of analog signal from water level sensor and temperature sensor to the 2:1 multiplexer that is designed is almost equal to the voltage level of the output signal of the buffer circuit, that is 1.6 volts. So there is no voltage drop, in other words, amplification of the buffer circuit is equal to 1 . The buffer circuit designed is to maintain thevoltage level of the multiplexer output in order that the voltage does notget dropped or voltage drop.

\section{REFERENCES}

[1] Yang Hong, Chi Fat Chan, JianpingGuo, Yuen Sum Ng, Weiwei Shi, Lai Kan Leung, Ka Nang Leung, "Design of Passive UHF RFID Tagi In 130nm CMOS Technology”, Circuits and Systems, 2008. APCCAS 2008.IEEE Asia Pacific Conference on, Macao.

[2] Muralikkrishna , UVM-based verification methodology for RFIDenabled smart-sensor systems, Analog Integrated Circuits and Signal ProcessingJanuary 2014, Volume 78, Issue 1, pp 191-207

[3] T. Keny, "Sensor Fundamental", in Sensor Technology Handbook,Elsevier,Eds. Jon S. Wilson, UK, Elsevier, 2005, pp. 12-15.

[4] Siclair, Ian R., "Sensors and Transducers", third edition, ButterworthHeinemann, 2001, pp.11

[5] IEEE1451 committee. IEEE Standard 1451.2-1997, Standard for a Smart Transducer Interface for Sensors and Actuators $\sim$ Transducer to Microprocessor CommunicarionProtocols and Transducer Electronic Data Sheet(TEDS)", Institute afElectrical and Electronics Engineen, Inc., Piscaraway, New Jeney 08855, September 26,1997

[6] Gupta, Arora, Singh, "Simulation and Analysis of 2:1 MultiplexerCircuits at 90nm Technology", International Journal of Modern Engineering Research (IJMER), Vol. 1, Issue 2, pp 642-646 